\title{
BALANCE DE LOS PROGRAMAS DE MEJORAMIENTO BARRIAL EN VENEZUELA. DE LA ERRADICACIÓN A LA HABILITACIÓN FÍSICA
}

\section{Elfide Mariela Rivas Gómez}

Es arquitecta, magíster Scientiae en Gestión Urbana. Fue gerente del programa de Habilitación Física de Barrios del Consejo Nacional de la Vivienda. Miembro fundador y activo del Centro de Barrios Comunitas. Centro Gumilla. Profesora del Departamento de Planificación Urbana. Carrera de Urbanismo, Universidad Simón Bolivar. Caracas.elmarivas@usb.ve 
BALANCE de LOS PROGRAMAS DE MEJORAMIENTO BARRIAL EN VENEZUELA

\title{
Resumen
}

Conocer y entender la evolución de las políticas urbanas de atención a la problemática de los barrios, cuáles son, cómo se han ejecutado, qué impacto ha tenido su aplicación, su desarrollo y consolidación y hacia dónde vamos en cuanto a su solución, es nuestro principal aporte.

El período de revisión se plantea desde 1960 a 2006, con énfasis en los últimos 25 años. En resumen, el balance supone que superada la política de desalojo y reemplazo de viviendas, a partir de los setenta la estrategia ha estado signada por la aplicación de programas de "mejoramiento urbano, consolidación y equipamiento", que han sido catalogados por Fernández Wagner (2003) como políticas habitacionales de segunda y tercera generación, programas de pequeña escala, con involucramiento de los pobladores y posteriormente avanzado los años noventa, programas de mayor alcance promovidos por las agencias internacionales que se inscriben en la Reforma del Estado, respectivamente.

\begin{abstract}
To know and to understand the evolution from the urban policies of attention to the problem of the shanty towns, which are, how they have been executed, what impact it has had its application, its development and consolidation and towards where we go as for its solution, it is our main contribution.

The period of review appears from 1960 to 2006, with emphasis in the last 25 years. In summary, the balance supposes that overcome the policy of eviction and replacement of housings, from the 70's the plan of the evolution of most of the slums, had been signed by the application of programs of "urban improvement, consolidation and equipment," that have been catalogued by Fernandez Wagner (2003) as urban policies of the second and third generation, programs of small scale, with involvement of the settlers and later advanced the Nineties, programs with greater reach promoted by the international agencies that register in the Reform of the State, respectively.
\end{abstract}




\section{Elfide Mariela Rivas Gómez}

BALANCE DE LOS PROGRAMAS DE MEJORAMIENTO BARRIAL EN VENEZUELA

\section{INTRODUCCIÓN}

América Latina es un territorio mayoritariamente urbano, según la ONU, desde 2000, el 90\% de la población de bajos y muy bajos ingresos reside en ciudades. Más de 170 millones de personas viven en pobreza en áreas urbanas y, para el caso venezolano, según el informe sobre Desarrollo Humano 2005 del PNUD, alrededor del 90\% de la población se ha radicado en centros urbanos, y de esa población hoy habitan en barrios ${ }^{1}$ más de 12 millones de personas (Informe sobre el Derecho a la Vivienda y Hábitat Dignos en Venezuela, PROVEA 2005).

La imperfección en el funcionamiento del mercado de la tierra y de la construcción de viviendas, los problemas estructurales a nivel político, social y económico, la radicación de la pobreza en nuestros países aunados a la incapacidad del Estado en desarrollar políticas de tierras y planes urbanos más adaptados a la realidad y menos discriminatorios, y en ofertar eficientes programas para cubrir la demanda habitacional, entre otras razones, han permitido la segregación urbana, el alojamiento desordenado e ineficiente de la ciudad, ocasionando sin duda, el origen de los asentamientos autoproducidos, los cuales se desarrollaron sin criterios de planificación, sin atender a los requerimientos de un proyecto urbanístico y sin la asistencia técnica adecuada.

Dar cuenta de esta realidad en el caso venezolano y cómo ha sido la evolución de las políticas y programas de atención al origen, evolución y desarrollo de los barrios, a través de un recorrido histórico y analítico, apuntando principalmente al período comprendido entre 1990 y 2006 es el principal aporte de este artículo.

\section{POLÍTICAS HABITACIONALES Y PLANIFICACIÓN URBANA: CÓMO LOS BARRIOS OCUPARONLACIUDAD}

La adquisición de una vivienda con las condiciones necesarias para vivir dignamente y la facilidad que el Estado genere para tal fin constituyen un derecho social que tanto la Constitución Nacional de 1961 como la de 1999 lo han consagrado como precepto fundamental.
1- En Venezuela se les llama barrios a los asentamientos informales o autoproducidos. 
2- www.derechos.org.ve publicaciones/infanual/ $\underline{2004 \_05}$

3- www.viviendaenred.com/ opinion/ Situación\%20Habitacional.pps

4- www.cvc.com.ve/download CEVIHAB.pdf
Aunque resulta notable el hecho de que la Constitución de la República Bolivariana de Venezuela (1999) incorpora el derecho a la participación de las comunidades en el desarrollo e implementación de los programas de vivienda (art. 70 y 184).

Sin embargo, a pesar de la existencia de un marco constitucional favorable y la derivación de éste en leyes, políticas y programas que han colocado el tema de la vivienda y los barrios en primer plano, se continúan desarrollando los barrios existentes y siguen surgiendo nuevos asentamientos urbanos, ausentes de planificación y carentes de estándares de urbanización, sin la adecuada intervención de las instituciones públicas competentes. Resulta evidente la incapacidad del Estado en la ejecución de políticas públicas eficientes y cónsonas que respondan al problema del hábitat popular.

\section{El Déficit de Viviendas}

Hacerse una idea del déficit habitacional en Venezuela no es una tarea fácil. No existe una data confiable y con la que se cuenta es contradictoria. No existe un informe del déficit habitacional emanado por alguna instancia gubernamental. La fuente oficial es un documento no publicado a la fecha, elaborado por el Instituto Nacional de Estadística (INE) y el Consejo Nacional de la Vivienda llamado "estimación de necesidades de atención habitacional" (Mimeo) de abril 2005, fundamentado en el Censo de Población y Vivienda de 2001, que se ha dado a conocer como una referencia en el balance de vivienda realizado desde el enfoque de los Derechos Humanos por el Programa Venezolano de Educación-Acción en Derechos Humanos (PROVEA)².

Otras referencias podemos ubicarlas desde la visión de La Fundación Vivienda Popular (FVP), Organización No Gubernamental en el área de vivienda ${ }^{3}$ y de La Cámara Venezolana de la Construcción (CVC) aglutinador de las empresas privadas de la construcción ${ }^{4}$. Veamos en el cuadro l la estimación del déficit habitacional según los diferentes entes descritos: 


\section{Elfide Mariela Rivas Gómez}

BALANCE DE LOS PROGRAMAS DE MEJORAMIENTO BARRIAL EN VENEZUELA

\section{Cuadro 1 Estimación del déficit habitacional}

\begin{tabular}{l|lll}
\hline Atención habitacional & INE & FVP & CVC \\
\hline Nuevas viviendas y mejoramiento de viviendas & 2.944 .374 & 2.899 .041 & 2.600 .000 \\
\hline $\begin{array}{l}\text { Atención cuantitativa: déficit bruto: } \\
\text { producción de nuevas viviendas por déficit } \\
\text { funcional (atención crecimiento vegetativo y } \\
\text { por hacinamiento) y la nuevas unidades ne- } \\
\text { cesarias por déficit estructural (aquellas que } \\
\text { deben ser sustituidas por deficiencia estruc- } \\
\text { tural de la edificación existente o por estar } \\
\text { ubicada en zona de riesgo) }\end{array}$ & & & \\
& & & \\
\hline $\begin{array}{l}\text { Atención cualitativa: adecuación, } \\
\text { mejoramiento de viviendas }\end{array}$ & 2.380 .008 & & \\
\end{tabular}

Fuente: informe sobre el derecho a la vivienda y hábitat dignos en Venezuela, PROVEA 2005

Se deslinda de estas cifras que la marcada discrepancia en los cálculos, en parte, tiene que ver con el concepto diferenciado de atención habitacional que cada una de las agencias maneja. En la literatura oficialista, se plantea la atención integral del hábitat, la necesidad de cubrir el déficit de urbanización y calidad de la vivienda en las zonas de barrios, además de la construcción de nuevos urbanismos especialmente para estratos de escasos recursos. Se valora, teóricamente, el patrimonio construido de manera progresiva por los pobladores, por lo cual se estima que se puede atender parte del déficit con la entrega de créditos para la mejora o crecimiento de la vivienda. Por su lado, la CVC enfatiza en la construcción de nuevas unidades más que la refacción o ampliación de la vivienda, asume más bien que el déficit debe ser atendido con la sustitución de la unidad habitacional existente por una nueva vivienda. 


\section{CUADER RSPACIO,CULTURA,SOCLEDAD}

BALANCE de LOS PROGRAMAS DE MEJORAMIENTO BARRIAL EN VENEZUELA

\section{La producción de viviendas}

Los datos censales de 1990 arrojaron que en Venezuela existían 3.889.819 viviendas mientras que el censo de 2001 registró 6.242 .516 viviendas. Esto significa que en once años se erigieron en Venezuela 2.352.697 nuevas unidades habitacionales. En ese mismo período, según El Plan Nacional de Vivienda 2003, el sector formal, Estado y empresa privada construyeron 715.749 viviendas, lo que indica que "el sector informal" autoconstruyó al mismo tiempo más del doble (70\%) (PROVEA, 2005). (Fotos 1 y 2)

\section{Mapa de políticas habitacionales}

Como ayuda pedagógica y expresión sucinta del proceso de actuación del Estado en materia urbana, describiremos a continuación las políticas urbanas de atención a la problemática de los barrios, apoyados en el trabajo de Cilento y Fossi (1998) titulado "Políticas de Vivienda y Desarrollo Urbano en Venezuela", en documentos oficiales y legislación de la República Bolivariana de Venezuela, así como investigación de campo en los últimos años.

Foto 1: Vista de Caracas por Yuraima Martín.

Foto 2: Barrio La Charneca por Mariela Rivas.
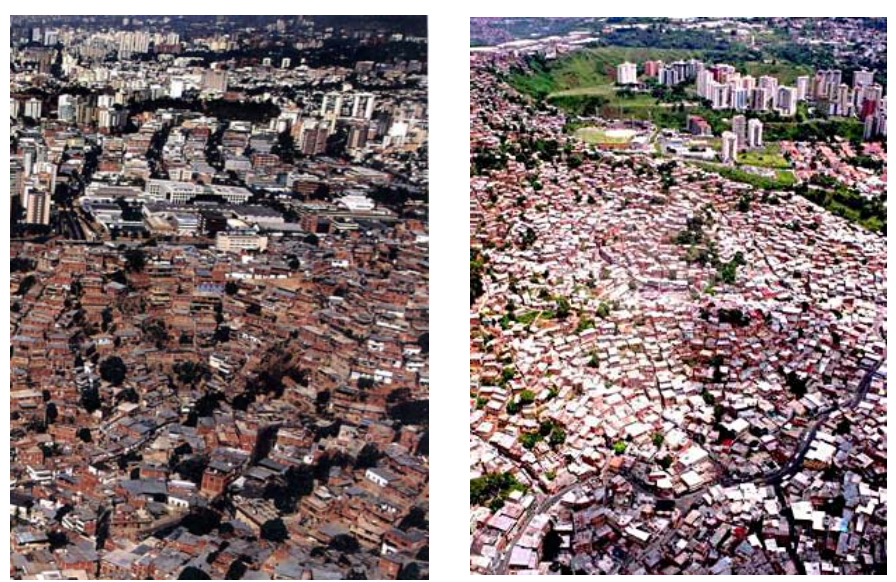

Entre los años veinte y cincuenta del siglo XX, las experiencias estuvieron signadas por el saneamiento y la renovación, se destaca como primera experiencia en la ciudad capital la reurbanización de El Silencio, proyecto del Arq. Carlos Raúl Villanueva que consistió en el saneamiento entre 1941 y 1944 de una barriada altamente degradada ubicada en el centro 


\section{Elfide Mariela Rivas Gómez}

BALANCE DE LOS PROGRAMAS DE MEJORAMIENTO BARRIAL EN VENEZUELA

de la ciudad donde existían 42 prostíbulos, 32 expendios de licores y habitaban 3.100 personas en 49 casas de vecindad. (De Sola, Ricardo 1988: 53). Posteriormente, se plantea el Primer Plan Nacional de Vivienda (1946-1949), se construyen los Superbloques (Banco Obrero, 1952-1957) y se ejecutan durante la dictadura de Pérez Jiménez desalojos masivos en Caracas.

A partir de 1958, posterior a la caída del gobierno dictatorial, se inicia un proceso hasta hoy día irreversible de invasiones, la producción de barrios urbanos pobres se constituye en la forma de ocupación residencial del espacio urbano más importante del país por parte de los estratos sociales menos favorecidos.

En las décadas de los sesenta y setenta del siglo pasado, a pesar de las recomendaciones de los expertos en el área y de las Comisiones de Urbanismo, el Estado se niega a instituir una política pública de adquisición de tierras. Las zonas de barrios para la época se estimaban en un $20 \%$.

En los años ochenta se deterioraron los indicadores generales de calidad del entorno urbano, medidos a través del acceso a los servicios básicos; asimismo los centros urbanos llegan a concentrar cerca del $85 \%$ de la población total del país; población que en su mayoría se ve forzada a alojarse en zonas de barrios, llegando a estimarse su ocupación en un $45 \%$.

Ya para los años noventa, las zonas de barrios sobrepasan el 50\% de ocupación territorial urbana. Aun cuando el Estado, invertía cuantiosas sumas de dinero en infraestructura y equipamientos, los barrios seguían caracterizándose por ser sectores no urbanizados.

Veamos en el cuadro 2 los aspectos más relevantes de esta década y los acontecimientos que se suceden con la nueva institucionalidad a partir de la instauración del gobierno bolivariano presidido por Hugo Chávez. 


\begin{tabular}{|c|c|}
\hline Año & Descripción \\
\hline Los noventa & 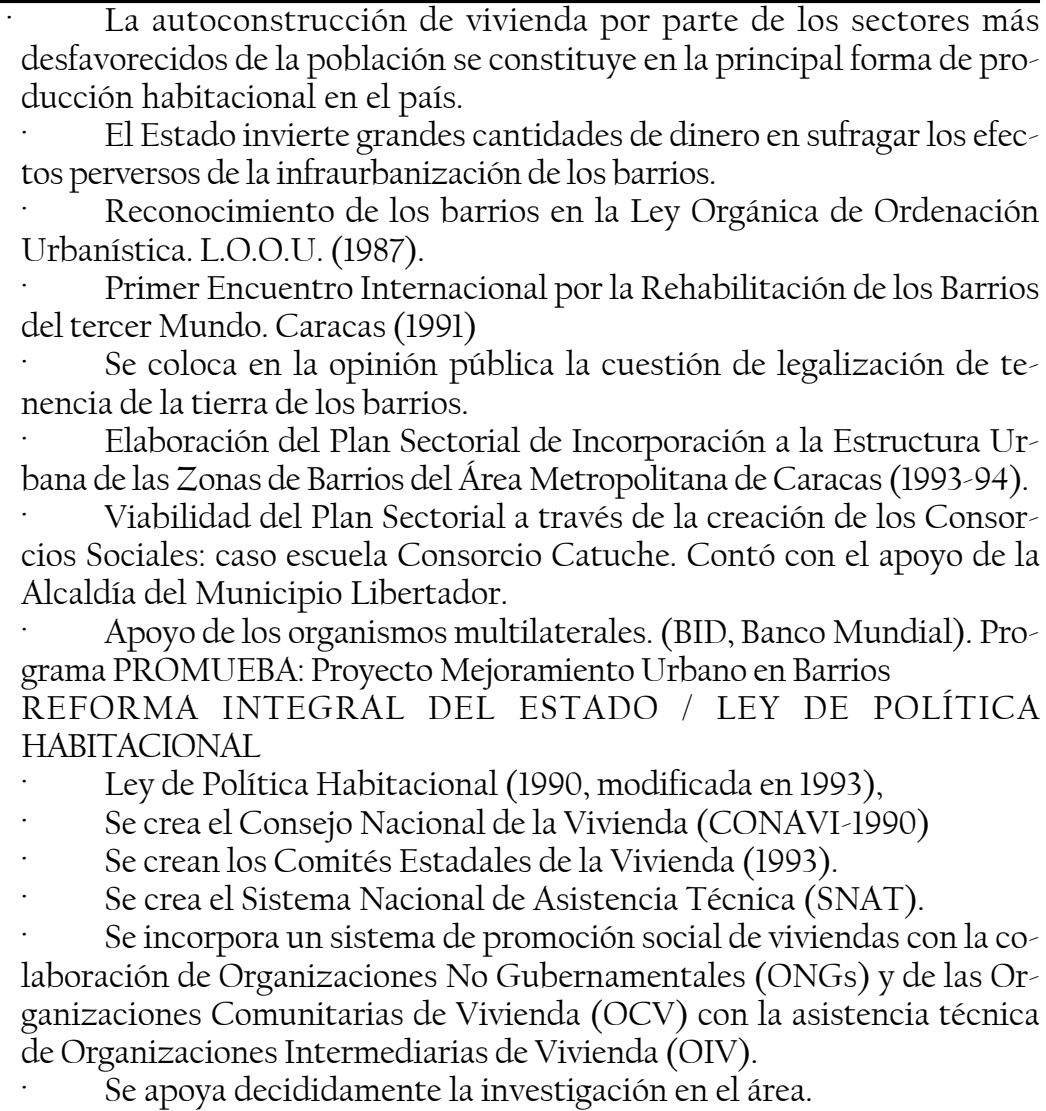 \\
\hline
\end{tabular}




\section{Elfide Mariela Rivas Gómez}

BALANCE DE LOS PROGRAMAS DE MEJORAMIENTO BARRIAL EN VENEZUELA

A las puertas LEY ORGÁNICA DE SEGURIDAD SOCIAL/ PROYECTOS DE LACOPRE del milenio

* $\quad$ Aprobación de la Ley Orgánica de Seguridad Social (1997), que establece un nuevo Sistema de Seguridad Social, en el cual se considera, entre otros sectores, el de la vivienda. A tal efecto se inicia la modificación de la L.P.H. (junio 1998).

\section{9}

\section{DECRETO CON RANGO DE LEY QUE REGULA EL SUBSISTEMA DE VIVIENDA Y POLÍTICA HABITACIONAL}

* Reestructuración del CONAVI y a finales de 1999 se aprueba el decreto con rango de ley que regula el subsistema de vivienda y política habitacional. Se diseña la implementación de seis programas, a saber:

1. Atención a Pobladores de la Calle: niños, ancianos e indigentes

2. Habilitación Física de las Zonas de Barrios

3. Mejoramiento y Ampliación de Casas en Barrios y Urbanizaciones Populares

4. Rehabilitación de Urbanizaciones Populares y Cascos Tradicionales

5. Nuevas Urbanizaciones y Viviendas de Desarrollo Progresivo.

6. Viviendas Regulares (Decreto que Regula el Subsistema de Vivienda y Política Habitacional, 1999).

* Caracas Mejoramiento de Barrios (Cameba 1999-2004): proyecto cofinanciado por el Banco Mundial que busca incorporar a la estructura planificada de la ciudad de Caracas a unos 200 mil habitantes de las Unidades de Diseño Urbano ${ }^{5}$ UDU 10- La Vega y UDU 4- Petare Norte. (FUNDACOMUN: http://www.fundacomun.gov.ve/proyectos).

* Tragedia Nacional en diez estados del país por lluvias torrenciales y deslaves: Vargas y Distrito Capital los más afectados.

2000

* Entre 1999 y el 2000 se invierte más de 100 millardos de bolívares (142 millones de dólares) en (1) pre-inversión: estudios, proyectos para unidades de diseño urbano, UDU, (conjunto de barrios), planes especiales de ordenamiento para Unidades de Planificación Física, UPF (grandes zonas de
5- En la metodología de los Planes Sectoriales se plantea entre una de sus primeras fases el "establecimiento y la delimitación precisa de las zonas relativamente grandes $y$ complejas que se han

denominado Unidades de

Planificación Física. Están constituidas por agregados de barrios, por lo general continuos espacialmente, que pueden dividirse en varias áreas equivalentes a urbanizaciones, o a etapas de desarrollo de urbanizaciones...; es decir, que cada Unidad de Planificación Física puede dividirse en varias Unidades de Diseño Urbano." Villanueva y Baldó (1998:25). A partir de allí se da una clasificación de otro tipo de zonas como Unidades de Diseño Urbano Separadas (UDUA), Unidades Aisladas (UA), Unidades Separadas (US), de acuerdo con características espaciales-geográficas,

demográficas, tamaño, etc. (Conavi, 2000) 
barrios) o casos emblemáticos, cartografía y (2) inversión: obras en casos pilotos y emergencias.

$2001 \quad * \quad$ Desde 2000, las diferentes instituciones nacionales relacionadas con el área de vivienda con funciones específicas de planificación, construcción de viviendas, banco de tierras, apoyo a las comunidades, etc., dan un viraje a su razón de ser, todas se dedican a la construcción o mejoramiento de viviendas, creando una anarquía institucional que no logró efectivamente la ejecución masiva de viviendas.

* Inicio de la peregrinación de las autoridades en el CONAVI, promedio de un año de gestión por cada uno de los presidentes de la institución.

* Inicio del descalabro del CONAVI: interrupción de sus políticas y programas. Se deja de invertir gran cantidad de recursos presupuestados en el área

* Constituyente de la Vivienda: ningún resultado de impacto en el área que mostrar

2002-2003 * Continuarían desarrollándose por inercia pero sin claridad de acción hasta 2005 los programas habitacionales instituidos en el Decreto con Rango de Ley que Regula el Subsistema de Vivienda y Política Habitacional * Decreto 1666 del 4 de febrero de 2002: se dicta el "decreto mediante el cual se inicia el Proceso de Regularización de la Tenencia de la Tierra en los Asentamientos Urbanos Populares". Gaceta Oficial 37.378 del 4 de febrero de 2002)

\begin{tabular}{ll}
\hline 2004 & Se crea el Ministerio de Vivienda y Hábitat \\
$*$ & Se constituye la Misión Hábitat \\
$*$ & Se constituyen entre 2002 y 2004 cerca de 5.000 Comités de Tierra \\
& Urbana (célula política del sector vivienda en las zonas de ba- \\
& rrios) \\
& En Convenio entre el Ministerio de Vivienda y Hábitat y la Funda-
\end{tabular}




\section{Elfide Mariela Rivas Gómez}

BALANCE DE LOS PROGRAMAS DE MEJORAMIENTO BARRIAL EN VENEZUELA

ción AMHABITAT se lanza el Programa de Transformación Endógena de Barrios (sin apoyo político), con la finalidad de rescatar el Programa de Habilitación Física de Barrios. Caso emblemático: UPF 1 Ojo de Agua. Carretera vieja a la Guaira.

* En paralelo al programa de Transformación Endógena de Barrios se inicia con gran apoyo político el Programa de Transformación del Hábitat, a través de los Centros de Participación para la Transformación del Hábitat (CPTH). Se plantean como un estadio avanzado del CTU, nuevas responsabilidades y ampliación del radio de trabajo y acción

\begin{tabular}{l}
\hline 2005 * Interrupción por cambios de autoridades el programa de Transfor- \\
mación de Barrios, pero se continua apoyando técnica y financieramente a \\
los CPTH y CTU \\
* Se decreta el inicio de la liquidación de todos los organismos de \\
vivienda a escala nacional. \\
* Aprobación de la nueva Ley del Régimen Prestacional de Vivienda \\
y Hábitat
\end{tabular}

2006 * A principios de 2006 se crea la Fundación Misión Hábitat y Plan Caracas. GO 38.367 del 27 de enero. El plan consistirá en la "transformación física y estructural del área metropolitana de Caracas, la densificación de los centros urbanos y la construcción de nuevas ciudades... El Plan Caracas "prevé la jerarquización de las zonas según los niveles de vulnerabilidad, ejecutando la rehabilitación de la infraestructura básica de servicios y equipamientos colectivos así como la mejora y ampliación o construcción de viviendas". http://www.eluniversal.com/2006/01/31/eco art 3120lA.shtml * Se continua apoyando técnica y financieramente a los CPTH y CTU * Se plantea una nueva misión el área de Vivienda: Misión Villanueva que consistirá en la construcción de ciudades intermedias, micro ciudades y micro comunidades para resolver el problema de la vivienda.

Fuente: elaboración propia a partir de documentos oficiales, prensa escrita, páginas web oficiales de los organismos públicos y legislación venezolana. 
BALANCE DE LOS PROGRAMAS DE MEJORAMIENTO BARRIAL EN VENEZUELA

\section{ROL DEL ESTADO. LA GESTIÓN URBANA EN LAS ZONAS DE BARRIOS}

1. Nudos críticos generados por el Estado en su tarea de enfrentar la problemática urbana de los barrios.

\section{1.Centralismo y respuesta anacrónica del Estado}

La concepción y práctica de la planificación urbana en Venezuela ha estado signada por una postura ideológica estado-céntrica, sustentada en esquemas centralizados, de toma de decisiones netamente verticales y desestimando los aportes de otros sectores de la sociedad. Desde el punto de vista técnico-instrumental se ha traducido en el riguroso ajuste a métodos y no a realidades.

Obviamente, las actuaciones estatales que se generan de un sistema jerarquizado de planes de articulación vertical, vinculante en su contenido, producen, por un lado, la estandarización de los patrones de ocupación del territorio, y por el otro, se convierten en un obstáculo del proceso de democratización de las políticas urbanas.

Será a partir de los años cincuenta del siglo XX, cuando en Venezuela se inicia formalmente la planificación urbana a través de Planes Reguladores, posteriormente Planes Rectores hasta llegar en los años ' 80 a los Planes de Desarrollo Urbano Local (PDUL). Pero, lamentablemente estos planes contribuyeron a la segregación espacial, al fraccionamiento de la ciudad, con zonificaciones donde se privilegiaba la ubicación de las áreas residenciales de los sectores pudientes en relación con la prestación de equipamientos, servicios, calidad ambiental y paisajística versus el destino de la vivienda obrera relacionada más con la zonificación industrial y las periferias. Planes en donde el suelo ocupado por los barrios pobres era zonificado como área verde o área a reubicar, y será a partir de 1987 cuando se reconozca en la Ley Orgánica de Ordenación Urbanística los barrios.

Con la disposición legal el PDUL terminaba ignorando grandes piezas urbanas, que en algunos casos conformaban la mayor parte del territorio de la ciudad como el caso de la ciudad de Maracaibo, así las zonas de barrios serían objeto de Planes Especiales que no se implementarían por falta de una política pública que lo promoviera. 


\section{Elfide Mariela Rivas Gómez}

BALANCE DE LOS PROGRAMAS DE MEJORAMIENTO BARRIAL EN VENEZUELA

\subsection{Los errores de la planificación: la institucionalización de políticas perversas}

Los nuevos habitantes urbanos han ideado soluciones autoproduciendo su hábitat y ocupando, desafortunadamente en muchos casos, terrenos inapropiados. Esto sucede en parte porque el Estado, como hemos venido anunciando, no ha generado una política de tierras para desarrollar urbanizaciones populares. Cilento y Fossi (1998) refieren que en 1964, se crea la Comisión de Desarrollo Urbano y la Vivienda de la que surgió un informe conocido como Lander que fue desestimado y enunciaba: "El desordenado crecimiento de los centros urbanos, unido a la ausencia de una política de tierras donde construir viviendas de los tipos y precios que reclama la demanda potencial, se convierte en un 'cuello de botella', que dificulta hasta lo imposible la solución racional del problema de la vivienda" (Cilento, 1996 en Ob.Cit: 28).

En 1975, de nuevo una Comisión Presidencial recomienda poner en marcha urgentemente un programa de adquisición pública de tierras, para lo cual se crea el Fondo del Desarrollo Urbano (Fondur), pero sin dotación de los recursos económicos necesarios. Los escasos fondos entregados debieron ser destinados en buena parte, a la estabilización del mercado secundario de cédulas hipotecarias (Ob.Cit.). Institución que a partir de 1999 se convierte en otro organismo ejecutor de vivienda a la par del Instituto Nacional de Vivienda (Inavi), complicando tremendamente el panorama de desorganización del sistema de vivienda en Venezuela.

En contraposición y paradójicamente el Estado ha resultado tradicionalmente, en gran medida, el propulsor de las "invasiones" y del desorden urbano desde las etapas iniciales de conformación de los barrios en Venezuela. Afirma Pérez (1992), que el Estado tenía una doble cara: "Por una parte protegía la propiedad privada del suelo y además regulaba el tipo de edificaciones que podían construirse. Por el otro, personas muy vinculadas con el aparato estatal organizaban las invasiones para que surgieran construcciones en violación del derecho de propiedad..." (Ob.Cit: 92).

Aunados a esta problemática, otros sectores - sin descartar el propio Estado- durante mucho tiempo han apostado por la eliminación, demolición y sustitución de este tipo de 


\section{CUADERNO}

BALANCE DE LOS PROGRAMAS DE MEJORAMIENTO BARRIAL EN VENEZUELA

6- Este Plan Sectorial, coordinado por los arquitectos urbanistas Josefina Baldó y

Federico Villanueva, fue reconocido en 1995 con el Premio Nacional de Vivienda.

Tuvo sus antecedentes en dos experiencias: 1) Taller Vivienda de la Facultad de Arquitectura y Urbanismo de la Universidad Central de Venezuela, coordinado por el arq. Federico Villanueva que adopta para los proyectos de diseño urbano en barrios los métodos $y$

procedimientos de evaluación de urbanizaciones desarrollados para unos 25 países desde 1965 del programa Urban Settlement Desing in Developing Countries de la Escuela de Arquitectura y Planificación del Instituto Tecnológico de Massachussets y 2) El Primer Encuentro de Rehabilitación de Barrios del

Tercer Mundo realizado en

Caracas en 1991, en donde el enfoque de la intervención integral de los barrios es sostenido por representantes de más de 50 países y que ha sido compilado en 1995 por Josefina Baldó y Teolinda Bolívar en el libro La Cuestión de los Barrios. asentamientos, significando — según esta concepción — además de una tarea de "profilaxis social", la "limpieza urbana" que daría paso al desarrollo armónico de la ciudad formal.

\subsection{Evolución e involución de las políticas urbanas: un problema de voluntad política}

El Consejo Nacional de la Vivienda diseña en 1999 dentro de las políticas de vivienda la Habilitación Física de Zonas Barrios, como el programa que recogerá e implementará la experiencia del Plan Sectorial de Incorporación a la Estructura Urbana de las Zonas de los Barrios del Área Metropolitana de Caracas ${ }^{6}$ (Conavi, 1999: 3-7). Y no habrían pasado dos años después del inicio de esta política cuando por cambio de autoridades en 2001 se interrumpe el desarrollo de los proyectos por la misma institución rectora. ¿Cuántas expectativas centradas en respuestas integrales, visibles y concretas se frustran? Todo esto sucede cuando apenas se comenzaba a implementar en 1999 el Decreto con Rango de Ley que Regula el Subsistema de Vivienda y Política Habitacional y sus Normas de Operación.

Esta ley, en relación con sus antecesoras, da un viraje importantísimo; de un paradigma viviendista, fisicalista, estadístico, se pasa a un paradigma de sistema urbano que enarbola una visión integral del hábitat y se enfoca en saldar una deuda social con los excluidos, pues entiende la vivienda como un problema de la ciudad en su conjunto, la que necesitamos construir y la que necesariamente requiere ser reordenada y habilitada, atendiendo las necesidades diferenciadas de los habitantes a través de distintos programas habitacionales. Programas que van desde el cobijo a niños de la calle, ancianos e indígenas; en los que se habilitan barrios y rehabilitan cascos tradicionales y urbanizaciones populares; se mejora y amplía viviendas existentes hasta la generación de nuevos urbanismos, con participación de profesionales asociados a las comunidades involucradas y entregando, cuando así se requiera y se demuestre las capacidades, los recursos económicos por medio de lo que se denominó "administración delegada".

\section{Los programas de mejoramiento barrial en la agenda urbana}

El balance supone que superada la política de desalojo y reemplazo de viviendas, a partir de los setenta la estrategia, ha estado signada por la aplicación de programas de "mejoramiento urbano, consolidación y equipamiento", que han sido catalogados por Fernández Wagner 


\section{Elfide Mariela Rivas Gómez}

BALANCE DE LOS PROGRAMAS DE MEJORAMIENTO BARRIAL EN VENEZUELA

(2003) como políticas habitacionales de segunda y tercera generación, programas de pequeña escala, con involucramiento de los pobladores y posteriormente avanzado los años noventa, programas de mayor alcance promovidos por las agencias internacionales que se inscriben en la Reforma del Estado, respectivamente.

"El llamado mejoramiento urbano de barrios acepta que la existencia de zonas de pobreza es un problema estructural que en el fondo no puede ser resuelto. Por lo tanto, se plantea el objetivo de 'aliviar' la situación a través del mejoramiento de las condiciones de vida. La construcción de obras de infraestructura y de equipamientos comunales para lograr el objetivo no persigue necesariamente la integración definitiva de la zona de barrios a la ciudad. El enfoque de consolidación de barrios enfatiza el grado de dureza alcanzado por las viviendas en el barrio, y una dotación mínima de servicios básicos, aunque no necesariamente al mismo nivel del resto de la ciudad. La consolidación no contempla necesariamente las acciones para la regularización de la tenencia de la tierra, ni incluye iniciativas para apoyar y fortalecer los procesos de organización comunitaria." (Conavi, 2000). Foto 3 y 4

En definitiva y en la práctica, las políticas de Estado desde el Plan de Emergencia de 1958 - creado con la finalidad de otorgar salarios a la fuerza laboral, empleándola en la construcción de pequeñas obras como escaleras de acceso, pavimentación de veredas y creación de plazoletas en los barrios situados en cerros y/o bordeando quebradas (Diccionario de Historia de Venezuela, 1997) — hasta la evolución e institucionalización de los llamados programas de Consolidación y Equipamiento de Barrios y sus modalidades, han

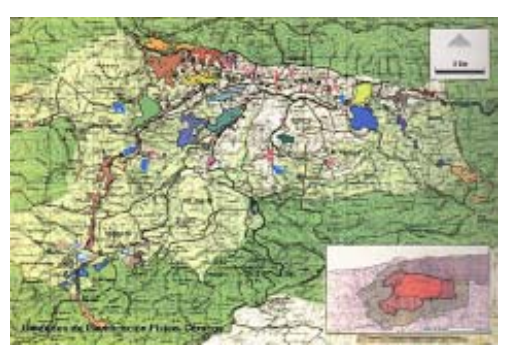

Imagen 1: Localización de las zonas de barrios de Caracas en el Plan Sectorial
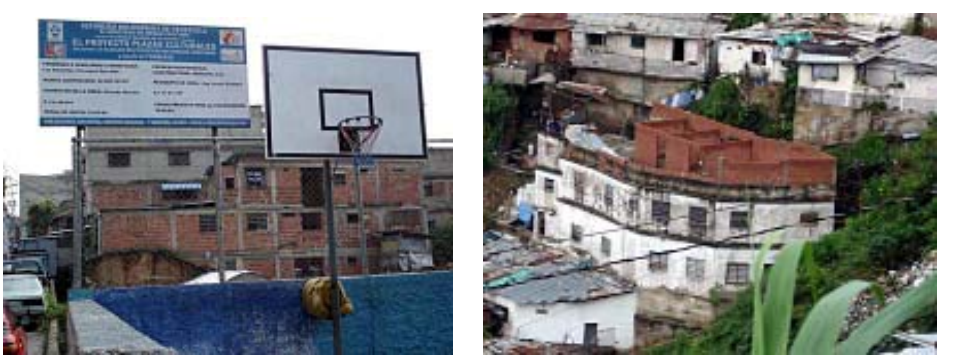

Foto 3 y 4: Barrio El Guarataro por Jesús Gómez. 
fracasado puesto que sólo han dado respuestas puntuales y coyunturales, por ser intervenciones parciales, "epidérmicas", carentes de proyectos que solucionen los problemas urbanos de manera integral.

\subsection{Política general de vivienda del gobierno bolivariano}

La puesta en vigencia del Decreto con Rango de Ley que Regula el Subsistema de Vivienda y Política Habitacional (1999) da cuenta de las posibilidades de cambios estructurales de la realidad antes expuesta. Se define una nueva etapa en la concepción, práctica y administración del problema de la vivienda en Venezuela. Según documentos oficiales del Conavi, el Estado asume la responsabilidad de saldar la deuda social acumulada de un $50 \%$ de la población de más bajos ingresos, que ha tenido que autoproducir su hábitat sin ningún tipo de asistencia técnica.

Bajo esta premisa, las autoridades del Conavi, basadas en el aprendizaje, rendimiento económico, factibilidad técnica y desarrollo social que han arrojado algunas de las experiencias de los Consorcios Sociales iniciados desde hace unos doce años en la ciudad de Caracas, junto con los Planes Sectoriales realizados en la Zona Capital y el eje Puerto La Cruz-Barcelona y a la experiencia social acumulada de sectores de la sociedad civil que han incursionado en el área, instrumenta el Programa II: Habilitación Física de las Zonas de Barrios y el Programa III: Mejoramiento y Ampliación de Casas en Barrios, como los nuevos programas que regirán la política urbana de atención a los barrios a partir de marzo de 1999. Se propone:

“... Proyectar y construir adecuadas infraestructuras locales de vialidad y servicios, suficientes equipamientos comunales, viviendas que sustituyan a las afectadas por alto riesgo y por las propias construcciones de habilitación física, organizando la actual ocupación del espacio hasta alcanzar, para los residentes, el mismo nivel de urbanización del resto de los venezolanos. También, en una escala mayor, la habilitación física consiste en proyectar y construir las necesarias obras de infraestructuras y equipamientos, que integren adecuadamente las grandes zonas compuestas por grupos de barrios con el resto de la ciudad. 


\section{Elfide Mariela Rivas Gómez}

BALANCE DE LOS PROGRAMAS DE MEJORAMIENTO BARRIAL EN VENEZUELA

Las prácticas de habilitación física en progreso enseñan que la combinación de proyectos profesionalmente excelentes, con la dirección en manos de las comunidades beneficiadas, no sólo garantiza la calidad de las obras materiales realizadas, sino su apropiación y adecuada utilización por los habitantes de los barrios, en un proceso de desarrollo simultáneo de la ciudad y ciudadanía, es decir, de la educación colectiva en la convivencia y la negociación en aras del bien común. Esto es lo fundamental, para no mencionar el rendimiento inusitado de las inversiones, la transparencia administrativa, la calificación y desarrollo de microempresas locales, o la facilitación de todo tipo de proyectos colectivos en lo educacional, sanitario y cultural" (Conavi, 1999: 4,5).

Las debilidades de esta política parecieran estar, más que en su concepción, en la burocracia estatal y en la paralización propiciada por algunas de las autoridades que asumieron responsabilidades en el Conavi, representando para los 246 casos iniciados en el período entre 1999 y 2000, que comenzaban a tener éxito al menos en la organización comunitaria y en el desarrollo de los proyectos ${ }^{7}$, razón por la cual Venezuela no tiene vitrina construida. Si comparamos, por ejemplo con la experiencia argentina de la provincia de Buenos Aires, similar en su concepción, el subprograma de Urbanización de Villas Miseria, en menos de dos años puede, aun con las críticas que se le puedan atribuir, mostrar resultados concretos en obra construida.

Ante la paralización propiciada desde el Conavi ${ }^{8}$, algunos organismos ejecutores de viviendas regionales y municipales y las propias comunidades han asumido independientemente y amparado en el marco legal dar continuidad a sus proyectos. Ejemplo de ello, la siempre luchadora comunidad de Catuche llegó al extremo de presentar, basados en el derecho a la vivienda y la participación, un recurso de amparo constitucional contra el Conavi, el cual les favoreció, ordenándose la ejecución forzosa.

En relación con el Programa II: Habilitación Física de Barrios, la propuesta de organización de la comunidad para desarrollar el proyecto colectivo de ordenamiento de su barrio, estuvo dirigido en una primera etapa, a la promoción de figuras organizativas jurídicas, asociaciones civiles independientes, con personalidad jurídica, compuesta por representantes de la comunidad, técnicos del área social y del área urbana, de la arquitectura,
7- Algunas de estas comunidades relatan sus experiencias relacionadas con este Programa promovido desde el Conavi en la compilación realizada por la Fundación Vivienda Popular en el libro Construyendo

Comunidades, 20 Experiencias Venezolanas, año 2000.

\author{
8- La interrupción de políticas \\ o programas sin la debida y \\ responsable evaluación ha \\ perjudicado enormemente al \\ gobierno del presidente Chávez, \\ como comenta Martha \\ Valmitjana (2002:127) no se \\ puede dar bandazos en cuanto \\ a la política a seguir o detener \\ programas en marcha sin \\ proponer alternativas. En el \\ caso del Programa II de \\ Habilitación Física en Zonas \\ de Barrios, Conavi pareciera \\ mantener serias reservas. \\ Puede ser que éste comulgue \\ con sus lineamientos generales, \\ pero quizás no en la forma \\ operativa de llevarlo a la \\ práctica; al respecto, ha \\ congelado los recursos \\ necesarios de algunos \\ proyectos en marcha, sin dar \\ alternativas".
}


ingeniería y afines, con capacidad técnica y legal para gestionar junto a los entes gubernamentales los proyectos. Como contribución al desarrollo de esta política, que comenzó teniendo éxito y una excelente receptividad en todos los actores, especialmente en las 246 comunidades que se incorporaron, y que compartimos tanto conceptualmente como en la práctica profesional, vale reunir las críticas y reflexiones que han surgido a posteriori sobre el programa en su corta implementación:

1. La movilización de una política de corte nacional, cuya presión gubernamental se traduce en cuántos proyectos se ejecutan, cuánto dinero se compromete, generó la masificación sin permitir en muchos casos reinterpretar las necesidades locales.

2. La capacitación, formación y sensibilización de los profesionales participantes no se cumplió a plenitud, salvando el trabajo realizado por un buen número de profesionales, muchos por desconocimiento y otros con intenciones perversas, obviaron el principio que se intentaba fomentar en primer orden, un trabajo dialógico, donde comunidad y equipo profesional están al mismo nivel construyendo un proyecto colectivo.

3. Cualquier política pública que no contemple la fase de seguimiento y evaluación, está condenada al fracaso; es el caso que para la primera fase de implementación del Programa II, aún previsto y propuesto un sistema de Evaluación Participativo, éste como ya indicamos en enero 2001 fue interrumpido.

Pareciera que los cambios de política que experimentan continuamente los gobiernos de turno, y en el caso del actual gobierno que, aun manteniéndose en el poder desde 1999, ha presentado en la entidad rectora del área de vivienda un director por año, cada uno de ellos con ideas, planes y proyectos nuevos y heterogéneos moviendo los cuadros técnicos y las políticas constantemente. Veamos en el cuadro 3 en la descripción de los programas de mejoramiento barrial en el período 1999-2006 la discontinuidad y los virajes de política (Ver cuadro 3).

Esto nos lleva a plantearnos iserá entonces la situación descrita en el manejo de las políticas de vivienda en este período, después de haberse planteado un cambio radical en el enfoque de atención habitacional y una mirada integral hacia el hábitat, otra involución 


\section{Elfide Mariela Rivas Gómez}

BALANCE DE LOS PROGRAMAS DE MEJORAMIENTO BARRIAL EN VENEZUELA

Cuadro 3: desarrollo de los programas de mejoramiento barrial en el período 1999-2006

\begin{tabular}{|c|c|c|}
\hline Año & Programa & Descripción \\
\hline $1999-2000$ & $\begin{array}{l}\text { Programa Nacional de } \\
\text { Habilitación Fisica } \\
\text { de Zonas de Barrios }\end{array}$ & $\begin{array}{l}\text { Instituido en el Decreto Ley que Regula el Sistema de Vivienda y } \\
\text { Politica Habitacional y sus respectivas Normas de Operación. Desde el punto de } \\
\text { vista metodológico desarrolla las etapas que componen un proyecto urbano: } \\
\text { diagnóstico (análisis de sitio), propuesta urbana, anteproyecto urbano y } \\
\text { anteproyectos y proyectos de obras, bajo un sistema de planificación y gestión en el } \\
\text { tiempo de las obras y proyectos, con participación de la comunidad organizada y técnicos. } \\
\text { Se iniciaron anteproyectos urbanos para } 250 \text { zonas de barrios del pais con la idea } \\
\text { de urbanizarlos progresivamente. Se interrumpió }\end{array}$ \\
\hline 2002 & $\begin{array}{l}\text { Regularización de la } \\
\text { Tenencia de la Tierra } \\
\text { en los Asentamientos } \\
\text { Urbanos Populares. } \\
\text { Decreto } 1666\end{array}$ & $\begin{array}{l}\text { Masiva constitución de Comités de Tierras Urbanas: se ha logrado } \\
\text { organizar a sectores de la comunidad para realizar catastros comunitarios } \\
\text { para lograr la regularización de la tenencia de la tierra en sectores } \\
\text { populares, especialmente barrios. Continúa. Esta politica deberia ser } \\
\text { complementaria a la de ordenación fisica de las zonas de barrios }\end{array}$ \\
\hline 2004 & $\begin{array}{l}\text { Programa de } \\
\text { Transformación } \\
\text { Endogena de Barrios } \\
\text { Reedición del Programa } \\
\text { de Habilitación Fisica } \\
\end{array}$ & $\begin{array}{l}\text { Se inventariaron cerca de } 300 \text { proyectos de habilitación física. Se logró } \\
\text { desarrollar alrededor de } 130 \text { Planes Maestros de Obras Fisicas y Sociales } \\
\text { para ejecutar los anteproyectos de habilitación física desarrollados en el } \\
\text { período } 2000-2001 \text {. Se Interrumpió }\end{array}$ \\
\hline $\begin{array}{l}2004 \\
2006\end{array}$ & $\begin{array}{l}\text { Programa de } \\
\text { Transformación de } \\
\text { Hábitat Popular } \\
\text { Programa de carácter } \\
\text { político-partidista, cuyo fin } \\
\text { planteado desde sus inicios } \\
\text { fue constituir un } \\
\text { movimiento de } \\
\text { pobladores, apoyándose en } \\
\text { el perfil politico que tiene el } \\
\text { proceso de regularización } \\
\text { de la tenencia de la tierra }\end{array}$ & $\begin{array}{l}\text { Propuesta de los Comités de Tierra Urbana a la Misión Vivienda: la propuesta } \\
\text { es pasar de la regularización jurídica a la fisica. Se le dio un gran impulso en } 2005 \text {. } \\
\text { De los resultados que se pueden evaluar hasta el momento, se recoge que los } \\
\text { proyectos y trabajo de los CPTH no tienen desde el punto de vista metodologico } \\
\text { la visión integral y estructural de la relación espacio-sociedad y de la intervención } \\
\text { a la estructura y morfología del barrio para solventar el déficit de urbanización } \\
\text { de la zona. El trabajo ha consistido en la sustitución de ranchos por viviendas, mejoras } \\
\text { de viviendas y construcción de obras puntuales de mitigación de riesgos. Vale decir } \\
\text { una tarea loable pero desvinculada a la real posibilidad de ordenamiento del conjunto } \\
\text { urbano. Un balance positivo podría plantearse en relación con la participación de la } \\
\text { población desde sus bases, aunque también habría que analizar la mediación } \\
\text { politico-partidista. Actualmente en ejecución pero sin promoción de nuevos casos }\end{array}$ \\
\hline
\end{tabular}

Fuente: elaboración propia a partir de documentos oficiales, prensa escrita, páginas web oficiales de los organismos públicos. 
de las políticas habitacionales y urbanas en el tratamiento estructural de los barrios? o iestamos claramente, a pesar del discurso y los enunciados de una literatura oficial progresista en el área de vivienda, ante la continuidad de la inoperancia del aparato estatal, desconcertándonos ante la creencia de que se iniciaron cambios estructurales en la implementación de políticas públicas en el área urbana y habitacional en nuestro país?

\section{CONCLUSIONES}

Resulta contradictorio que a pesar del cúmulo de experiencias y de los esfuerzos estatales, la realidad que prime sea que nuestras ciudades sigan estando ocupadas por extensas áreas empobrecidas y con un gran déficit de urbanización. Hemos evidenciado el fracaso casi generalizado de los programas de mejoramiento urbano barrial diseñados e institucionalizados hasta el momento por el Estado venezolano. Veamos algunas de las razones, según mi criterio, para tan rotundo fracaso:

1. Estudios sobre el tratamiento, desarrollo y ordenamiento de los barrios han sido seriamente expuestos por investigadores y otros interesados en el tema, evidenciando que es un fenómeno urbano común en América Latina y que requiere urgente atención por quienes tienen a su cargo la planificación y gestión urbana de nuestras ciudades. Parece que nos hacen falta líderes con voluntad política que conduzcan estos procesos.

2. Apartando algunas casos basados en el Plan Sectorial mencionado, a contadas prácticas desarrolladas por el Conavi hasta 1998 a través del SNAT y alguna que otra experiencia de las políticas gubernamentales entre 1999 y 2006 y algún caso aislado de corte local, los programas de mejoramiento urbano barrial desarrollados por el Estado, han estado enmarcados desde la perspectiva de esquemas populistas, cortoplacistas e inmediatistas, e invirtieron grandes sumas de dinero en la consolidación de una infraestructura caótica, contribuyendo así al afianzamiento de la infraurbanización.

En lo político, se han dado respuestas de acuerdo con los intereses de los funcionarios de turno; en lo gerencial y técnico, desde los entes competentes, no se ha logrado crear y diseñar propuestas y proyectos integrales para intervenir y habilitar los barrios, en parte, 


\section{Elfide Mariela Rivas Gómez}

BALANCE DE LOS PROGRAMAS DE MEJORAMIENTO BARRIAL EN VENEZUELA

por negligencia, apatía e incompetencia, y también por todo el aparataje burocrático; en lo social, en lo humano es donde se ha hecho sentir con mayor gravedad el problema: se considera a los habitantes de nuestros barrios ciudadanos de tercera. Foto 5

3. Si no se logra la voluntad política que permita continuar con los cambios esperados, seguiremos iniciando, cada vez, un nuevo proyecto, que no logrará en definitiva arrojar resultados que beneficien a las colectividades. Las políticas deben ser de carácter estatal y no gubernamental. Y en el caso del período gubernamental actual, las políticas no pueden seguir estando signadas por la discrecionalidad y ajustadas al pensamiento y método del director - presidente o ministro de turno. En siete años, la política de vivienda en Venezuela ha estado presidida por ocho administradores distintos.

Las políticas deben tener seguimiento y contar con evaluaciones técnicas y financieras, deben ser perfectibles y legitimadas por las comunidades para darles sostenibilidad, que garantice su continuidad y una cobertura importante.

4. Definitivamente quienes toman las decisiones a nivel político, desde la alta magistratura y los ocupantes de cargos de puestos claves en el área, no han entendido el problema de la vivienda y los mecanismos de atención habitacional para una Venezuela netamente urbana cuya población mayoritariamente está viviendo en zonas de barrios y produciendo anualmente más del $50 \%$ de las viviendas nuevas. Habilitación de tierras urbanas, apoyo económico y asistencia técnica para la autoconstrucción progresiva, disminuiría en gran medida las invasiones de terrenos, muchas veces no aptos para procurarse los sin techo un pedazo de hogar. Ordenamiento, urbanización, habilitación o transformación, más legalización con proyectos con la gente al frente y con la parti-

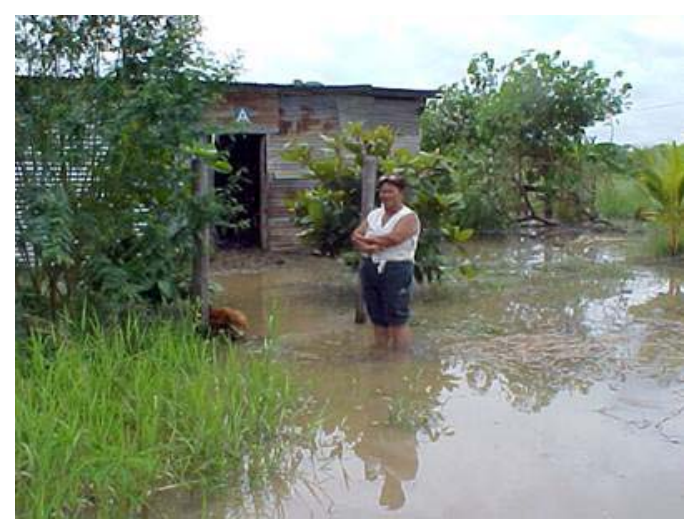

Foto 5: Barrio José Antonio Páez (Guanarito) por Mariela Rivas 
cipación de profesionales urgen para atajar unos trozos de ciudad que se están cayendo a pedazos. ¿Por qué no intentamos aprender de las lecciones, de la realidad urbana y de las estadísticas?

\section{BIBLIOGRAFÍA}

CILENTO, Alfredo y FOSSI, Víctor (1998) "Políticas de Vivienda y Desarrollo Urbano en Venezuela (1928-1997). Una cronología crítica". En Partidas, E y Arrieta, L (comp.) Conferencia Internacional de Urbanización y Vivienda, URVI 98. pp. 23-41 Barquisimeto: Tipografía y Litografía Horizonte.

DE SOLA, Ricardo (1988) "La Reurbanización de El Silencio. Crónica”. Instituto Nacional de la Vivienda.

FERNÁNDEZ WAGNER, Raúl (2003) "Los programas de mejoramiento urbano barrial en América Latina". En: http://www.urbared.ungs.edu.ar/debates foros.

PÉREZ, R. (1992) "Retorno a los Barrios. Repensando una investigación socio-jurídica quince años después" en Sociología del diritto (3): 89-102.

VALMITJANA, Martha (2002) "La planificación urbana en situaciones de urgencia social: las zonas de barrios". Rev. Venezolana de Economía y Ciencias Sociales, 2002, vol. 8, no 3 (septiembre-diciembre), pp. 119-129.

VILLANUEVA, Federico y BALDÓ, Josefina (1998) “Un Plan para los Barrios de Caracas: síntesis del Plan Sectorial de Incorporación a la Estructura Urbana de las Zonas de Barrios del Área Metropolitana de Caracas y de la Región Capital (Sector Panamericana y Los Teques)" Consejo Nacional de la Vivienda. Caracas: Impresión Minipres C. A. Premio Nacional de Investigación en Vivienda de 1995.

\section{OTRAS FUENTES}

Decreto mediante el cual se inicia el Proceso de Regularización de la Tenencia de la Tierra en los Asentamientos Urbanos Populares. Decreto 1666 del 4 de febrero de 2002. Presidencia de la República Bolivariana de Venezuela. http://www.escenariotributario.com/ 2002febrero.htm 


\section{Elfide Mariela Rivas Gómez}

BALANCE DE LOS PROGRAMAS DE MEJORAMIENTO BARRIAL EN VENEZUELA

Decreto que regula el Subsistema de Vivienda y Política Habitacional" GACETA OFICIAL 5392 (1999)

Diccionario de Historia de Venezuela. Caracas: Exlibris. FUNDACIÓN POLAR (1997)

Informe sobre el Derecho a la Vivienda y Hábitat Dignos en Venezuela www.derechos.org.ve/ publicaciones/infanual/2004 05 PROVEA (2005)

Ley De Política Habitacional, 1993. Gaceta Oficial 37.378 (2002)

Lineamientos Generales par la Instrumentación del Programa II: Habilitación Física delas Zonas de Barrios. Caracas: Consejo Nacional de la Vivienda CONAVI (2000)

Política de vivienda 1999-2004. Qué hacer y cómo hacerlo, en relación al problema de la vivienda. Caracas: Consejo Nacional de la Vivienda. CONAVI(1999)

Situación Habitacional en Venezuela. Caracas, 2004 (Mimeo) FUNDACIÓN VIVIENDA POPULAR (2004)

Unmundoen proceso de urbanización. Informemundial sobrelosasentamientoshumanos. 1996 Centro De Las Naciones Unidas Para Los Asentamientos Humanos (1996) Colombia: Tercer Mundo Editores. 
